\title{
Study of interior temperature distribution and implementation of smart materials in the truck cabin during summer conditions.
}

\begin{abstract}
Temperature in the inner part of the automotive vehicle compartment is very important to provide affluent state to the passengers. Temperature in the interior part of the chamber will be improve, when the automobile is parked right under neath the sunlight. The radiation episode to the surface varies from second to second based upon its geographical position (latitude and longitude of the place), orientation, season, time of the day and atmospheric conditions. Inadvertence of clouds, the daily average illumination for the Earth is nearly $230 \mathrm{~W} / \mathrm{m} 2$.Major 13 hottest cities in these four zones were considered to study interior temperature and heat contents in the truck cabin. The data of temperatures in these cities at the interval of 11 am to 2 pm were collected for month of March to Mid of June for 2010 to 2017, and average max temperature and Relative Humidity (RH) were identified for these years. These values are further used for the calculations of Zenith (altitude) angle, azimuth (longitudinal) angle, and Sky temperature. In the mathematical modelling, CFD analysis, and heat transfer mechanism equations for conduction, convention and radiation through vehicle body and window glasses is important to determine interior truck cabin temperature, distribution and heat, then changing the material of the truck depending upon the CFD analysis done. Materials such as thermo electric materials, phase change materials and solar cells are implemented with electrical integration system. This research proposes a new perspective that by implementing these materials reduce of cabin internal temperature to a larger extent can be done and increase the fuel efficiency during summer.All in all, it can be terminated that by switch of the materials is the best method in reducing the interior temperature inside the truck cabin.
\end{abstract}

Keyword : Thermal Analysis; CFD; Thermo electric materials; Phase change materials; Solar cells; Electrical integration system. 